\title{
Management of Malignant Biliary Obstruction
}

\author{
Jonathan M. Lorenz, MD¹ \\ ${ }^{1}$ Department of Radiology, The University of Chicago, Chicago, Illinois \\ Semin Intervent Radiol 2016;33:259-267
}

\begin{abstract}
Address for correspondence Jonathan M. Lorenz, MD, Department of Radiology, The University of Chicago, 5841 S. Maryland Avenue, MC2026, Chicago, IL 60637 (e-mail: jlorenz@radiology.bsd.uchicago.edu).
\end{abstract}

\author{
Abstract \\ Keywords \\ - biliary obstruction \\ - cancer \\ - percutaneous \\ drainage \\ - interventional \\ radiology
}

The diagnosis of malignant biliary obstruction combines the use of clinical evaluation, diagnostic imaging, tissue sampling, and minimally invasive options with the initial goal of identifying candidates for curative resection. The most common causes of obstruction are pancreatic adenocarcinoma and cholangiocarcinoma, and most cases are too advanced for surgical options. Interventional radiologists and gastroenterologists offer palliative options for biliary drainage such as plastic stents and catheters, bare metal stents, and covered stents. This article provides an updated review of options and outcomes for the management of malignant biliary obstruction.
Objectives: Upon completion of this article, the reader will be able to describe the interventional management of patients with malignant biliary obstruction.

Accreditation: This activity has been planned and implemented in accordance with the Essential Areas and Policies of the Accreditation Council for Continuing Medical Education (ACCME) through the joint providership of Tufts University School of Medicine (TUSM) and Thieme Medical Publishers, New York. TUSM is accredited by the ACCME to provide continuing medical education for physicians.

Credit: Tufts University School of Medicine designates this journal-based CME activity for a maximum of 1 AMA PRA Category 1 Credit $^{\mathrm{TM}}$. Physicians should claim only the credit commensurate with the extent of their participation in the activity.

Malignant biliary obstruction most commonly results from pancreatic adenocarcinoma and cholangiocarcinoma. Other etiologies include gallbladder carcinoma, hepatocellular carcinoma, lymphoma, and metastasis to regional solid organs and lymph nodes. ${ }^{1}$ Curative resection is the primary goal of diagnosis and management but rarely an option because of local invasion and distant metastases at the time of clinical presentation. In the absence of effective screening methods for early detection, palliative decompression of advanced malignant obstruction by biliary catheterization or stent placement will remain a key component of the management of these patients. The most effective strategy to manage malignant biliary obstruction combines a multidis- ciplinary team of medical oncologists, diagnostic radiologists, surgical oncologists, gastroenterologists, and interventional radiologists. Management may include medical, endoscopic, percutaneous, and surgical therapeutic options depending on the etiology and the chance for cure. This article reviews the diagnosis and management of malignant biliary obstruction.

\section{Diagnosis}

Malignant biliary obstruction usually presents with painless jaundice, pruritus, and much less commonly signs and symptoms of infectious cholangitis such as fever and leukocytosis. Other features include dark urine resulting from increased bilirubin content, loss of weight and appetite, nausea, and vomiting. A baseline assessment of coagulation status as well as hepatic and renal function is critical, as abnormalities may increase the risk of subsequent imaging and minimally invasive studies. Tools for the initial evaluation of obstructing jaundice include imaging modalities and endoscopic retrograde cholangiopancreatography (ERCP). The goals are distinction between benign and malignant strictures, guidance of tissue sampling, assessment of the extent of local disease, and exclusion of distant metastases.

Distinction between benign and malignant strictures is most commonly made by magnetic resonance cholangiopancreatography (MRCP), ERCP, and, occasionally, percutaneous transhepatic cholangiography (PTC). PTC remains a secondline option to failed or infeasible ERCP for diagnosis and access for biopsy because of potential bleeding complications
Issue Theme Biliary Interventions; Guest Editor, Thuong G. Van Ha, MD
Copyright (c) 2016 by Thieme Medical Publishers, Inc., 333 Seventh Avenue, New York, NY 10001, USA.

Tel: +1(212) 584-4662.
DOI http://dx.doi.org/ 10.1055/s-0036-1592330. ISSN 0739-9529. 
associated with transhepatic access. In terms of diagnosis, a prospective study by Corvera et al compared MRCP to computed tomography (CT), ERCP, and PTC for identifying and distinguishing malignant versus benign biliary strictures. ${ }^{2}$ MRCP was supported as a noninvasive alternative to ERCP and both studies were more sensitive and specific than CT. The sensitivity of both studies was $85 \%$, and the specificities of MRCP and ERCP were 71 and 75\%, respectively. Recent studies have demonstrated a sensitivity of MRCP exceeding $96 \%$ and a specificity of $85 \%$ for distinguishing benign from malignant biliary obstruction. ${ }^{3,4}$ The sensitivity and specificity of ERCP for the determination of benign versus malignant disease are increased further by the addition of endoscopic ultrasound (EUS) and the newer technique of intraductal ultrasound. ${ }^{5}$

To further hone the diagnosis of malignant biliary obstruction, guidance of tissue sampling is the next goal. For larger intra- or extrahepatic masses, CT- and EUS-guided biopsies are options, depending on the location. For lesions invading or intrinsic to the bile ducts, options to improve the sensitivity of ERCP and PTC include brush cytology, forceps biopsy, and core biopsy. While the reported sensitivity of brush cytology is typically at or below $50 \%$, specificity is $95 \%^{6}$; forceps or needle biopsy can augment brush cytology to further increase sensitivity and specificity to 70 and $100 \%$, respectively. ${ }^{4}$ Some interventional radiologists have used percutaneous transhepatic cholangioscopy to provide direct visualization of the lesion or stricture, increasing the reported sensitivity and specificity to greater than $95 \%{ }^{7}$

The next step is determination of local and distal disease for staging. This information is critical as it distinguishes resectable cases from those requiring palliative therapies such as biliary catheterization or stent placement. For this indication, CT and magnetic resonance imaging (MRI) offer complete, multiplanar, cross-sectional imaging beyond the local primary tumor. Both CT angiography and MR angiography can evaluate for resectability by ruling out invasion or involvement of regional arteries and veins. Additional options to evaluate local extent include EUS, intraductal ultrasound, and percutaneous cholangioscopy through a transhepatic sheath. Fluorodeoxyglucose-positron emission tomography (FDG-PET) has been used for characterization of intrahepatic lesions and staging of regional and distal metastases. ${ }^{2,8}$

\section{Management}

\section{Medical and Surgical Options}

Treatment options for malignant biliary obstruction include medical therapy, surgical resection with or without preoperative biliary drainage, endoscopic or percutaneous biliary drainage (PTBD) via plastic catheters and stents, and metal stent placement. In the absence of infection, medical or surgical options should be considered before palliative interventions. For some indications such as lymphoma, biliary obstruction can be treated with conservative medical management with the aim toward reduction of lymphomatous infiltration or lymph node enlargement. ${ }^{9}$ Temporary endoscopic biliary drainage (EBD), PTBD, and retrievable covered stents are better options than permanent, self-expanding metal stents (SEMS) if interval resolution of obstruction using conservative management is feasible. For other cases, curative resection is the primary goal, and resectability varies with the malignant etiology and the stage of disease. As interventional and surgical techniques have advanced, the pool of resectable candidates has increased. For example, hilar cholangiocarcinoma was formerly considered unresectable with an extremely poor prognosis, but in the late 1990s, the success of aggressive resection of the involved liver and hilar nodes was described. ${ }^{10}$ Nimura et al described a strategy of surgical resection after PTBD and/or portal vein embolization in 100 patients with hilar cholangiocarcinoma who underwent successful curative hepatectomy. ${ }^{11}$ The survival rates were unexpectedly high: 43 , 26 , and $19 \%$ at 3,5 , and 10 years, respectively. Sugiura et al combined extensive resection of the bile ducts with partial hepatectomy for hilar cholangiocarcinoma in 83 patients and achieved a $20 \%$ survival rate at 5 years. ${ }^{12}$

\section{Preoperative Biliary Drainage}

For candidates for resection, the strategy of preoperative EBD or PTBD using plastic stents and catheters remains a topic of controversy stemming from the fact that instrumentation of the biliary tree prior to operative resection carries of theoretical risk of postoperative wound infection and cholangitis. ${ }^{13}$ In some cases, preoperative drainage is clearly indicated for clinical signs of infection such as fever, leukocytosis, and hypotension. In other cases, the clinical presentation and the time interval to surgical resection may favor the decision for preoperative drainage. In support of preoperative drainage, Abdullah et al retrospectively reviewed 82 patients who underwent surgical resection and reconstruction for ampullary carcinoma. ${ }^{14}$ The incidence of wound infection was actually less in the preoperative drainage group than the non-preoperative drainage group (2.9 vs. $25.5 \%$ ). No other differences between the two groups were noted for all factors evaluated -including sepsis, intra-abdominal abscess, biliary leakage, and patient survival. Preoperative drainage is also likely indicated when resection of hilar cholangiocarcinoma is planned, particularly when portal vein embolization is performed, as it may take weeks to hypertrophy the future liver remnant; chronic obstruction can lead to atrophy of the affected liver. In such cases, PTBD has its advantages over EBD. In a comparative observation study, Kloek et al reviewed outcomes of two groups, those undergoing EBD and those undergoing PTBD prior to resection of hilar cholangiocarcinoma. For EBD and PTBD, technical success rates were 81 and $100 \%(p=0.20)$ and infectious complications were 48 and $9 \%(p<0.05)$, respectively. ${ }^{15}$ The EBD group required more interventions. Finally, in 30 of 115 patients, EBD was converted to PTBD because of failure of the endoscopic approach. Other benefits of preoperative biliary drainage have been reported in patients undergoing pancreatic head resection, including reduced perioperative bacteremia and bleeding, ${ }^{16}$ reduced postoperative hospitalization, and reduced postoperative morbidity. ${ }^{17}$

In other published data including a cohort observational study of 311 patients by Sewnath et al, preoperative biliary drainage for malignant biliary obstruction resulted in no demonstrable benefit in terms of surgical morbidity and 
mortality, ${ }^{18,19}$ and, in some studies, was implicated in an increase in overall postoperative complications ${ }^{20}$ and an increase in postoperative infectious complications in patients with proximal cholangiocarcinoma. ${ }^{21,22}$ The best application of preoperative drainage may rest on case-by-case clinical considerations.

\section{Endoscopic versus Percutaneous Biliary Drainage}

In the absence of a medical or surgical therapeutic option for malignant biliary obstruction, palliative drainage for inoperable malignant biliary obstruction is performed using either endoscopic or radiologic guidance. EBD is considered the best initial option for therapeutic intervention in most cases, as it avoids the complications of percutaneous, transhepatic access, especially in patients with coagulopathy or ascites, and it avoids the complications associated with prolonged external biliary drainage catheters. $^{13}$ In most cases, PTBD is used only if the endoscopic approach fails or is infeasible. Exceptions exist. PTBD is favored for segmental obstruction by intrahepatic tumors or for select patients with hilar cholangiocarcinoma. Lee et al evaluated outcomes of ERCP and PTBD approaches using the Bismuth classification. $^{23}$ For Bismuth I or II lesions, no difference in patency rates was observed after metal stent placement by either method. In cases with unilateral or bilateral extension into the intrahepatic ducts (Bismuth III or IV), the best results were achieved when the ERCP approach was used for Bismuth III lesions and the PTBD approach was used for Bismuth IV lesions. PTC is particularly helpful when ERCP fails to opacify a portion of the biliary tree excluded by an obstruction; ultrasound guidance of percutaneous puncture allows selective access of the excluded ducts. In general, both methods are standard therapeutic options for all levels and etiologies of malignant biliary obstruction and their application is often determined by clinical factors as well as local expertise.

\section{Endoscopic Biliary Drainage}

During the past 30 years, EBD has continued to gain favor over percutaneous techniques for malignant biliary obstruction. More recently, its applicability has increased because of EUS-guided, sharp puncture techniques that bypass the obstructed papilla. EUS guidance of biliary access has become increasingly available as skilled endoscopists take this technique beyond the confines of tertiary academic centers. In the future, one would expect endoscopic success rates to increase and referrals for PTBD to decrease. During ERCP for malignant biliary obstruction, when traditional transpapillary drainage fails, EUS-guided transgastric, transduodenal, and transhepatic puncture of the biliary system offers a second-line option prior to referral for PTBD. The feasibility of EUS-guided, sharp puncture of the biliary system was established in short, pilot series between 2007 and 2009. ${ }^{24-26}$ This approach is most applicable to pancreatic head malignancy and involves placement of plastic or covered metal stents across the newly established tract. Technical and clinical success rates of these limited studies range from 91 to $100 \% .^{24,26}$

Despite operator dependency and the added difficulty associated with local malignancy, the success of transpapillary access for malignant biliary obstruction during ERCP is comparable to the success of PTBD (see later). The most common and potentially serious complication of ERCP is pancreatitis from injury to or distention of the pancreatic duct. Pancreatitis occurs in approximately 5\% of ERCP procedures, but for complicated, high-risk patients such as those with malignant biliary obstruction, the risk of pancreatitis can exceed $15 \% .{ }^{27}$ Less common complications include endoscope-induced gastrointestinal perforation, biliary sepsis, and bleeding complications from sphincterotomy and EUSguided sharp puncture techniques.

\section{Percutaneous Biliary Drainage}

PTBD is still commonly required for failed EBD, particularly outside of tertiary academic centers, where advanced EUS expertise may be limited. Again, PTBD is a potential option for any case of biliary obstruction, but always first line for hilar Bismuth IV cholangiocarcinoma, permanent internal/external biliary catheterization in cases not amenable to metal stent placement or repeated endoscopic exchange of internal plastic stents (e.g., Roux-en-Y anatomy), and for recurrent malignant obstruction refractory to repeat metal stent placement as the final treatment option.

Contraindications to PTBD are relative and include coagulopathy and ascites. The timing and feasibility of corrective measures should be considered on a case-by-case basis, but consensus guidelines have been published. The Society of Interventional Radiology (SIR) standards of practice guidelines classify new PTBD as a high-risk procedure and recommend correction of international normalized ratio to less than 1.5, cessation or reversal of heparin for activated partial thromboplastin time greater than 1.5 times control, withholding of clopidogrel and aspirin for 5 days, and withholding of fractionated heparin for 24 hours or up to two doses prior to the procedure. $^{28}$ On occasion, PTBD may be necessary despite suboptimal or infeasible corrective measures. Preprocedural antibiotics are indicated for the prevention of infectious complications, ${ }^{29,30}$ and postprocedural antibiotics are considered on a case-by-case basis. When patients initially present with symptoms of cholangitis, resolution can be achieved with immediate PTBD followed by interval metal stent placement.

Published success rates of PTBD are comparable to those of EBD for malignant biliary obstruction. In 1987, Speer et al randomized 75 patients with malignant obstructive jaundice to endoscopic versus percutaneous drainage and found a higher success rate for endoscopic drainage (81 vs. $61 \%$, $p=0.017) .{ }^{31}$ However, those results were recently called into question. Leng et al in a meta-analysis of 264 screened articles and 3 randomized controlled trials comprising 183 cancer patients found no difference in success rate between PTBD and EBD when the article of Speer et al was included, but significantly greater therapeutic success of PTBD when the article of Speer et al was excluded. ${ }^{1}$ The 30-day mortality and complication rates were similar for the PTBD and the EBD groups. As EUS-guided sharp puncture techniques become more commonly used, the success of EBD is likely to increase. Based on published success rates, the SIR guidelines recommend a success threshold for PTC of $90 \%$, and a success threshold for accessing the small bowel of $90 \%{ }^{13}$ 
Major, procedure-related complications of PTC and PTBD occur in 4 to $7 \%$ of cases ${ }^{13,32-36}$ and include sepsis, cholangitis, cholecystitis, pancreatitis, pleural transgression with associated complications, tumor seeding, hemorrhage, and death. ${ }^{37,38}$ Patients with malignant biliary obstruction, particularly proximal obstruction, have higher complication rates from PTBD compared with patients with other indications, likely related to associated comorbidities and poor health status at the time of presentation. PTBD-related cholangitis after malignant biliary obstruction has a low rate of additional, related morbidity and mortality with appropriate treatment ${ }^{39}$ using catheter exchange and intravenous antibiotics. Frank sepsis occurs more commonly when PTBD is performed in patients with preexisting cholangitis and after prior ERCP. The latter results from preexisting bacterial seeding combined with contrast distention during PTC. After PTBD, paradoxical worsening of signs of biliary obstruction can indicate hemobilia resulting from biliary communication to hepatic arterial or venous structures or from disruption of tumor vascularity. Acute bleeding with hemodynamic changes usually results from arterial injury, and sources include chest wall arteries as well as hepatic arteries. ${ }^{35}$ CTA is often helpful prior to arterial coil embolization to identify focal active extravasation or pseudoaneurysm. For venous transgression or bleeding from tumor vascularity, catheter upsizing, catheter repositioning, or covered stent placement may be options.

If long-term drainage is necessary, delayed, catheter-related complications include clogging and dislodgment. ${ }^{13,40}$ Dislodgment can lead to sidehole placement in the hepatic vasculature or parenchyma, resulting in bleeding through the catheter lumen, gastrointestinal bleeding, and hemobilia causing obstruction of the biliary tree by clot. Options for treatment include catheter repositioning and replacement, catheter upsizing, and in rare cases of biliary-venous fistula, covered stent placement. ${ }^{41}$ When internal/external PTBD is not achieved (access to small bowel fails), an external PTBD may be necessary. Potential complications of prolonged external drainage of bile include electrolyte abnormalities and malnutrition related to poor intestinal absorption. Repeat attempts to access the small bowel for internal/external PTBD should be done in most cases.

\section{Stent Options and Goals}

The goals of stent placement for malignant biliary obstruction are palliation of symptoms and prevention of complications associated with disease progression. To these ends, stent patency should exceed patient lifespan whenever possible to minimize the need for repeat interventions. For example, for inoperable pancreatic adenocarcinoma, the median survival is 6 to 11 months, ${ }^{42}$ and for patients with metastatic disease, median survival drops to 2 to 6 months. ${ }^{43}$ Given these low survival rates, stents provide lifetime patency for most of these patients, and in most cases, SEMS are favored over plastic stents to limit the need for future endoscopic interventions. For malignant biliary obstruction, published data support SEMS over plastic stents for their higher clinical success rates, higher long-term patency rates, and lower cost because of the reduction of secondary procedures, ${ }^{44}$ even in the case of hilar cholangiocarcinoma as demonstrated by a short, prospective randomized trial of plastic versus metal stents. ${ }^{45}$

\section{Self-expanding Metal Stents}

High technical and clinical success rates of SEMS makes them well suited for palliative treatment of biliary obstruction caused by pancreatic carcinoma or cholangiocarcinoma, ${ }^{46}$ as patency rates closely match survival rates. In a retrospective, multicenter European study of 240 patients, Rossi et al reported 78 and $67 \%$ patency at 25 weeks for nitinol and stainless steel SEMS, respectively, in a patient population with 25 - and 50-week survival rates of 42 and $16 \%$, respectively. ${ }^{47}$ Even for the difficult scenario of hilar biliary obstruction, Freeman and Overby prospectively evaluated unilateral SEMS placement for 35 patients with hilar biliary obstruction and found a $77 \%$ clinical success and a median patency of 5.4 months. ${ }^{48}$ Other authors have achieved similar success with unilateral SEMS, Y-configured SEMS (- Fig. 1), and Y-configured covered SEMS (CSEMS) for hilar obstruction. ${ }^{49,50}$ When unilateral atrophy is present, stent placement into the most viable lobe may be preferential to bilateral $Y$ configuration of dual SEMS, unless infection of the atrophic biliary component is suspected.

Technical aspects of metal stent placement are best planned using preliminary MRCP or CT, particularly for hilar malignant obstruction. ${ }^{48}$ Considerations when performing metal stent placement include preserving the papilla if possible to avoid ascending cholangitis and to improve long-term patency, ${ }^{51,52}$ minimizing distention of the gallbladder to avoid postprocedure cholecystitis, and avoiding the caging of branch points, if possible, to avoid associated biliary obstruction. Prior to stent placement, balloonassisted clearance of sludge from the biliary tree may reveal a shorter segment of malignant involvement and allow placement of a shorter stent. This maneuver can prevent unnecessary extension of the stent above the hilum (-Fig. 2). If possible postdilatation is avoided as SEMS continue to expand after placement in most cases, and postdilatation can cause bleeding from tumor vascularity and obstruction from incursion of tumor through the interstices or around the edges of the stent.

\section{Bare versus Covered Stents}

While SEMS provide quality-of-life improvement via a catheter-free period of symptom relief, they may fail as a result of tumor overgrowth at the margins of stent placement or tumor in-growth across the interstices. ${ }^{23}$ As a result of the latter complication, investigators have reported improved long-term patency rates after CSEMS placement (-Fig. 2). Controversy remains regarding the value of CSEMS, as they add considerable cost to stent placement. On the surface, added durability would theoretically offset that cost. The long-term patency rates of CSEMS for malignant obstruction far exceed most currently published survival rates ${ }^{47,53-57}$; therefore, the widespread use of CSEMS may hinge on investigations showing resultant improvements in patient survival. 

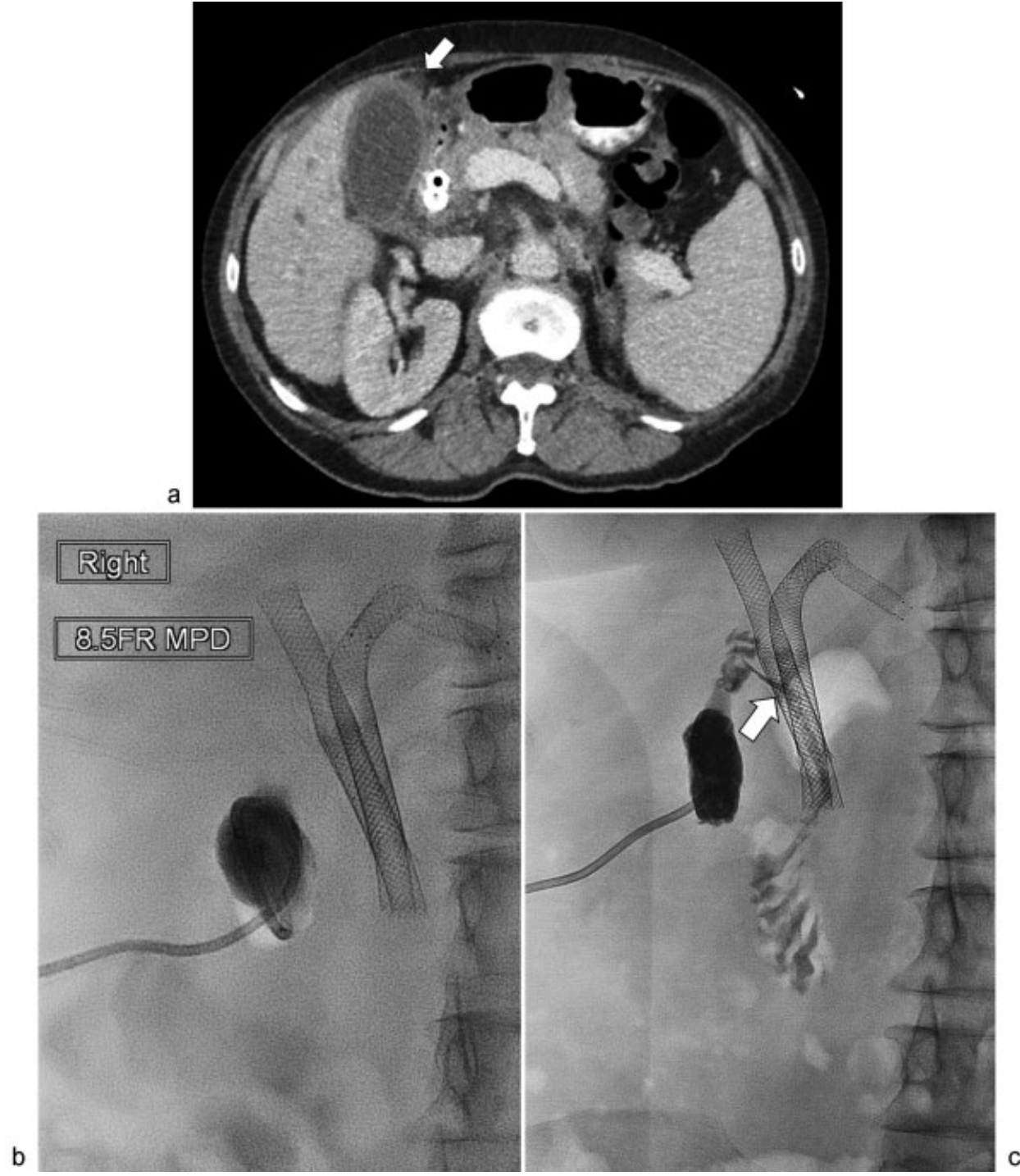

Fig. 1 (a) A 66-year-old woman with Bismuth IV cholangiocarcinoma treated with bilateral bare metal stents in a Y-configuration presents with right upper quadrant pain, fever, and leukocytosis. CT with contrast demonstrates gallbladder wall thickening and surrounding fat stranding (arrow) suspicious for acute cholecystitis. (b) Cholecystostomy catheter was placed. Contrast injection shows no free passage of contrast into the cystic duct. (c) Contrast injection after 1 month shows free passage of contrast from the gallbladder to the small bowel (arrow). The catheter was eventually removed with no recurrence of cholecystitis.

For example, in a cohort observational study of 80 consecutive patients undergoing expanded polytetrafluroethylene/fluorinated ethylene propylene (ePTFE/FEP)-covered metallic stent placement, Fanelli et al reported patency rates of 95.5, 92.6, and $85.7 \%$ at 3,6 , and 12 months, respectively, and survival rates of 40 and $20.2 \%$ at 6 and 12 months, respectively. ${ }^{54}$ These patency rates far exceed those reported for bare SEMS, resulting in the authors' conclusion that CSEMS are safe and highly effective, but the lack of concordance between patency and patient survival calls their cost-effectiveness into question.

Some studies have reported improved patient survival after CSEMS versus SEMS for malignant biliary obstruc$\operatorname{tion}^{47,57}$ and others have found no survival benefit. ${ }^{58}$ In a prospective, randomized trial of 80 patients comparing ePTFE/FEP-CSEMS to nitinol SEMS for pancreatic cancer, Krokidis et al reported a mean patency of 166 days versus 234 days $(p=0.007)$ and median survival of 203.2 versus
247 days $(p=0.06)$ for SEMS versus CSEMS, respectively. ${ }^{58}$ The authors noted comparable costs, particularly since CSEMS were associated with no tumor in-growth and required fewer secondary interventions; on the other hand, in a large, randomized multicenter trial of 400 patients with malignant biliary distal biliary obstruction, Kullman et al found no significant difference between CSEMS and SEMS in terms of patency rates or survival rates. ${ }^{59}$

\section{Investigational Options}

Drug-eluting SEMS offer the theoretical effect of suppression of tumor growth in addition to the prevention of direct tumor in-growth. Few studies exist, but results of short pilot studies are promising. Suk et al reviewed the application of a metallic stent covered with a paclitaxel-incorporated membrane for malignant biliary obstruction in a multicenter pilot study. ${ }^{60}$ Mean patency was 429 days and cumulative patency rates at 
a

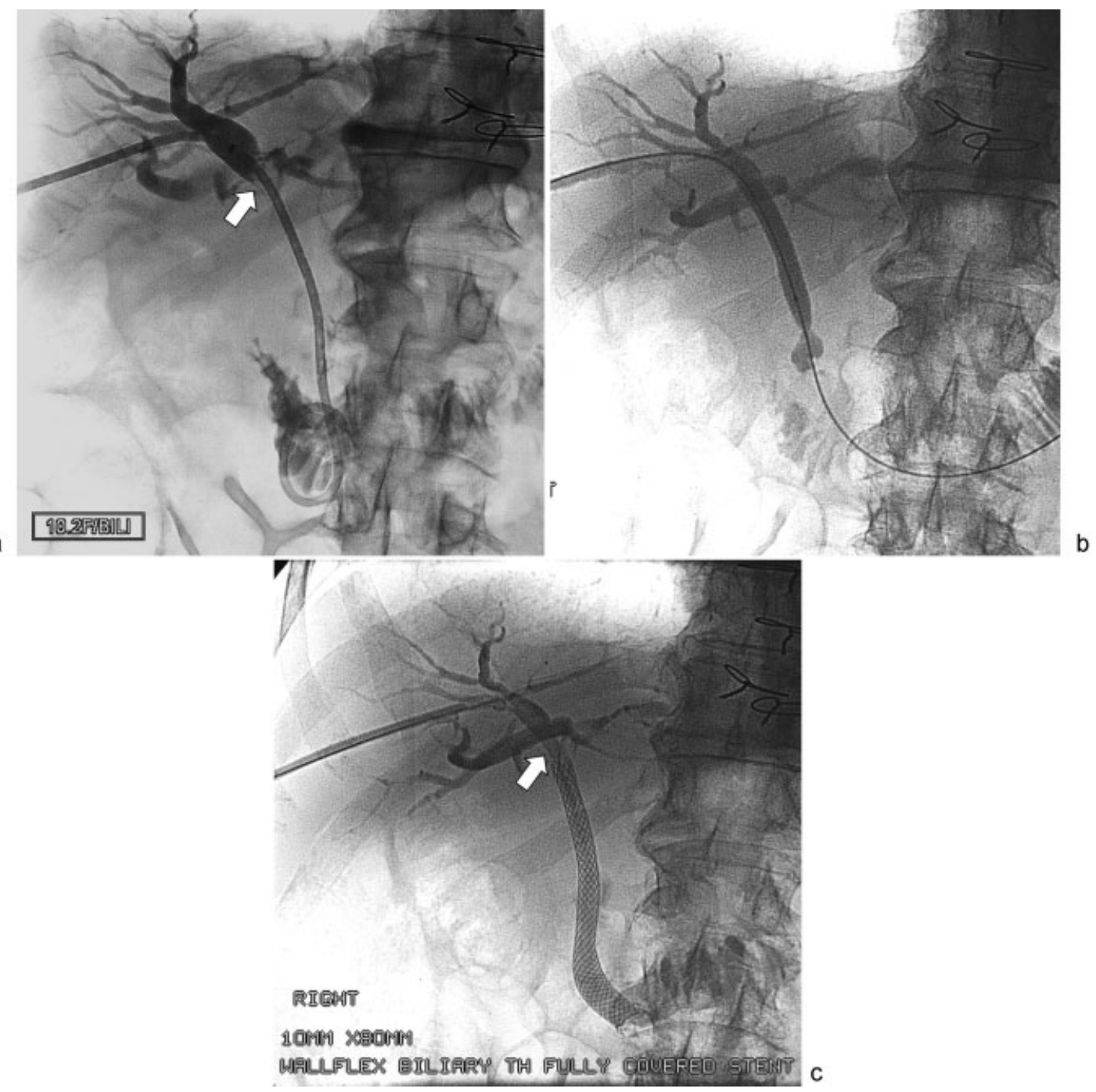

Fig. 2 (a) An 81-year-old man with locally invasive gastric cancer presents for metal stent placement after interval percutaneous drainage for cholangitis. Sludge in the biliary hilum gives the false appearance of malignant involvement (arrow). (b) Prior to stent placement, low-pressure balloon clearance and dilatation of the biliary tree were performed. (c) Involvement of the hilum was excluded by balloon clearance (arrow) and a covered metal stent was placed. Involvement of the sphincter of Oddi made transpapillary stent placement necessary in this case.

3, 6, and 12 months were 100,71 , and $36 \%$, respectively. Further studies focusing on survival benefit are warranted.

Percutaneous and endoscopic radiofrequency ablation has been used as a primary therapy to resolve malignant biliary strictures and obstruction before stent placement and as a secondary therapy for restenosis after stent placement. Preliminary results have been promising to improve long-term patency rates. ${ }^{61,62}$

\section{Complications of Metal Stents}

Complications of SEMS include cholangitis, cholecystitis, pancreatitis, migration, and obstruction. Cholangitis is typically well tolerated and treated with antibiotics and repeat intervention to clear stent obstruction, if necessary. Lee et al in a review of 134 patients comparing ERCP- and PTBDguided metal stent placement found lower rates of procedure-related cholangitis with PTBD. ${ }^{63}$ Measures to mitigate the risk of cholangitis include avoidance of overdistention of the biliary system with contrast material, suprapapillary rather than transpapillary SEMS placement if possible, avoidance of branch point obstruction by SEMS if possible, and treatment of cholangitis to resolution with temporary drainage and antibiotics prior to metal stent placement. Cholecystitis occurs in approximately $5 \%$ of cases of SEMS placement across the cystic duct ostium and is managed well by percutaneous cholecystostomy. Cholecystostomy catheters after SEMS placement may be removable after resolution of cholecystitis if patency of the cystic duct and biliary tree is demonstrated ( - Fig. 1). However, some cases of persistent obstruction of the cystic duct by tumor or stent, indefinite external drainage, or chemical ablation of the gallbladder may be necessary ${ }^{64}$ Authors have described increased rates of CSEMS versus SEMS migration ${ }^{59}$ ( - Fig. 3 ), but no significant difference in the rates of other complications, including cholecystitis and pancreatitis.

When metal stents fail, secondary EBD or PTBD procedures are required for additional stent placement, sludge or stone clearance using baskets, balloons, and endoscopic lithotripsy. 


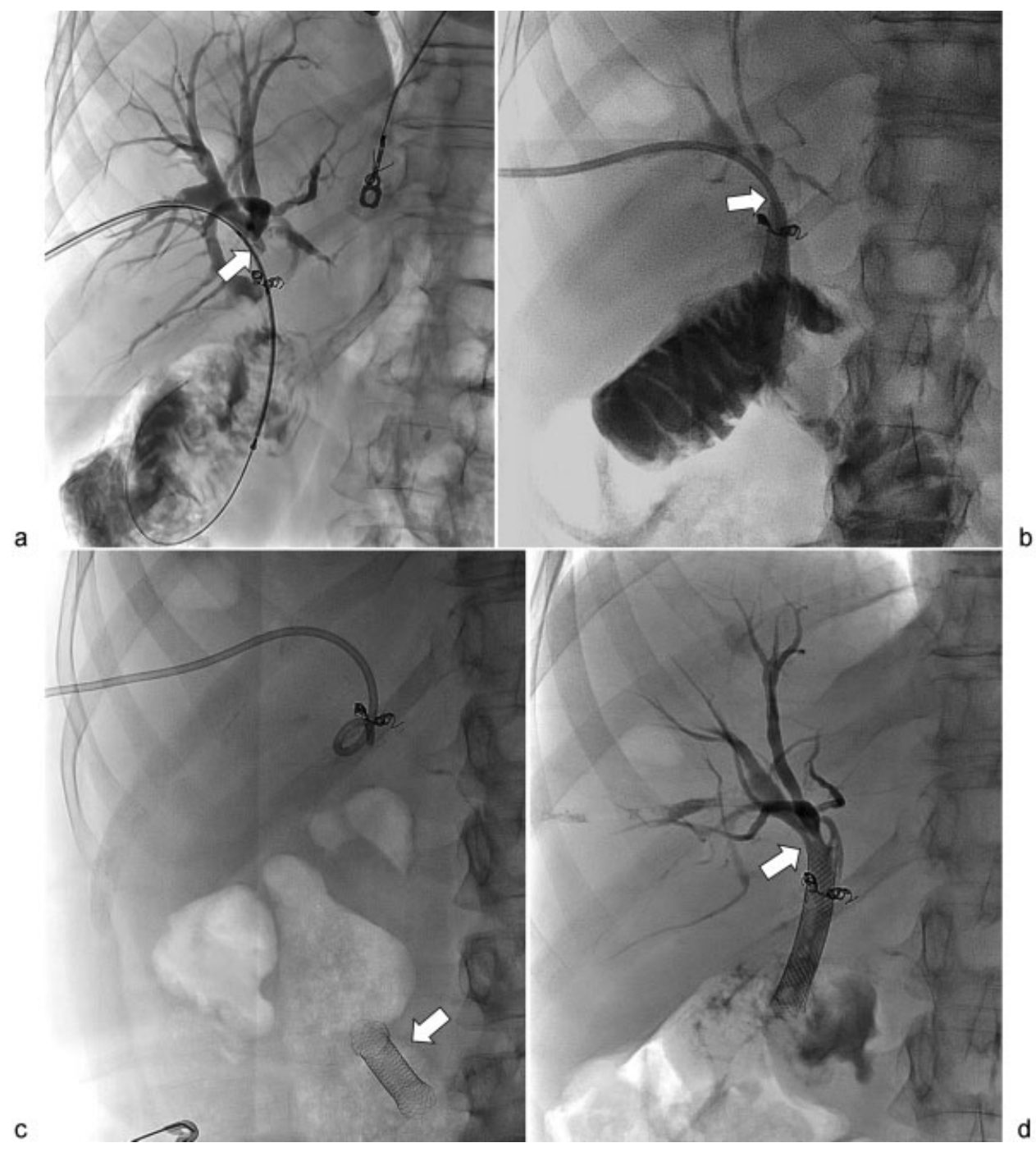

Fig. 3 (a) A 49-year-old woman who underwent a Whipple procedure for pancreatic cancer presents with recurrent biliary obstruction. A short common bile duct is anastomosed to a Roux-en-Y. Hepatic arterial coils are related to prior iatrogenic hemobilia. Obstruction extends to the hilum, and a nondeployed 4-cm-long covered stent is preliminarily placed with the proximal end above the obstruction (arrow). (b) After deployment, the proximal end has migrated distally (arrow), and most of the stent protrudes into the Roux limb. (c) Abdominal radiograph obtained the next day shows stent migration distally into the small bowel (arrow). (d) A 4-cm-long bare metal stent was placed with the proximal end flared and appropriately placed within the biliary hilum (arrow).

For patients with extended survival after metal stent placement, disease progression may be so extensive that repeat metal stent placement and other therapeutic measures become infeasible. In such cases, placement of a permanent internal/external PTBD may be required. ${ }^{63}$

\section{Conclusion}

The management of malignant biliary obstruction continues to evolve with the advancement of techniques offered by surgeons, gastroenterologists, and interventional radiologists. SEMS have improved the quality of life of nonresectable patients, and further investigation is warranted to establish survival benefits and efficacy of more recent advancements such as CSEMS and radiofrequency ablation. The best approach to malignant biliary obstruction is a multidisciplin- ary effort that combines skills and advancements to optimize outcomes.

\section{References}

1 Leng JJ, Zhang N, Dong JH. Percutaneous transhepatic and endoscopic biliary drainage for malignant biliary tract obstruction: a meta-analysis. World J Surg Oncol 2014;12(1):272

2 Corvera CU, Blumgart LH, Akhurst T, et al. 18F-fluorodeoxyglucose positron emission tomography influences management decisions in patients with biliary cancer. J Am Coll Surg 2008; 206(1):57-65

3 Suthar M, Purohit S, Bhargav V, Goyal P. Role of MRCP in differentiation of benign and malignant causes of biliary obstruction. J Clin Diagn Res 2015;9(11):TC08-TC12

4 Xu MM, Sethi A. Diagnosing biliary malignancy. Gastrointest Endosc Clin N Am 2015;25(4):677-690 
5 Canto MI, Hruban RH, Fishman EK, et al; American Cancer of the Pancreas Screening (CAPS) Consortium. Frequent detection of pancreatic lesions in asymptomatic high-risk individuals. Gastroenterology 2012;142(4):796-804, quiz e14-e15

6 Burnett AS, Calvert TJ, Chokshi RJ. Sensitivity of endoscopic retrograde cholangiopancreatography standard cytology: 10-y review of the literature. J Surg Res 2013;184(1):304-311

$7 \mathrm{Kim} \mathrm{EH}, \mathrm{Kim} \mathrm{HJ}$, Oh HC, et al. The usefulness of percutaneous transhepatic cholangioscopy for identifying malignancies in distal common [corrected] bile duct strictures. J Korean Med Sci 2008; 23(4):579-585

8 Kim JY, Kim MH, Lee TY, et al. Clinical role of 18F-FDG PET-CT in suspected and potentially operable cholangiocarcinoma: a prospective study compared with conventional imaging. Am J Gastroenterol 2008;103(5):1145-1151

9 Di Sena V, Thuler FP, Macedo EP, Paulo GA, Della Libera E, Ferrari AP. Obstructive jaundice secondary to bile duct involvement with Hodgkin's disease: a case report. Sao Paulo Med J 2005;123(1): 30-32

10 Kosuge T, Yamamoto J, Shimada K, Yamasaki S, Makuuchi M. Improved surgical results for hilar cholangiocarcinoma with procedures including major hepatic resection. Ann Surg 1999;230(5): 663-671

11 Nimura Y, Kamiya J, Kondo S, et al. Aggressive preoperative management and extended surgery for hilar cholangiocarcinoma: Nagoya experience. J Hepatobiliary Pancreat Surg 2000;7(2): 155-162

12 Sugiura Y, Nakamura S, Iida S, et al. Extensive resection of the bile ducts combined with liver resection for cancer of the main hepatic duct junction: a cooperative study of the Keio Bile Duct Cancer Study Group. Surgery 1994;115(4):445-451

13 Burke DR, Lewis CA, Cardella JF, et al; Society of Interventional Radiology Standards of Practice Committee. Quality improvement guidelines for percutaneous transhepatic cholangiography and biliary drainage. J Vasc Interv Radiol 2003;14(9 Pt 2, Suppl): S243-S246

14 Abdullah SA, Gupta T, Jaafar KA, Chung YF, Ooi LL, Mesenas SJ. Ampullary carcinoma: effect of preoperative biliary drainage on surgical outcome. World J Gastroenterol 2009;15(23):2908-2912

15 Kloek JJ, van der Gaag NA, Aziz Y, et al. Endoscopic and percutaneous preoperative biliary drainage in patients with suspected hilar cholangiocarcinoma. J Gastrointest Surg 2010;14(1):119-125

16 Lygidakis NJ, van der Heyde MN, Lubbers MJ. Evaluation of preoperative biliary drainage in the surgical management of pancreatic head carcinoma. Acta Chir Scand 1987;153(11-12): 665-668

17 Marcus SG, Dobryansky M, Shamamian P, et al. Endoscopic biliary drainage before pancreaticoduodenectomy for periampullary malignancies. J Clin Gastroenterol 1998;26(2):125-129

18 Sewnath ME, Birjmohun RS, Rauws EA, Huibregtse K, Obertop H, Gouma DJ. The effect of preoperative biliary drainage on postoperative complications after pancreaticoduodenectomy. J Am Coll Surg 2001;192(6):726-734

19 Hatfield AR, Tobias R, Terblanche J, et al. Preoperative external biliary drainage in obstructive jaundice. A prospective controlled clinical trial. Lancet 1982;2(8304):896-899

20 Povoski SP, Karpeh MS Jr, Conlon KC, Blumgart LH, Brennan MF. Association of preoperative biliary drainage with postoperative outcome following pancreaticoduodenectomy. Ann Surg 1999; 230(2):131-142

21 Hochwald SN, Burke EC, Jarnagin WR, Fong Y, Blumgart LH. Association of preoperative biliary stenting with increased postoperative infectious complications in proximal cholangiocarcinoma. Arch Surg 1999;134(3):261-266

22 Ferrero A, Lo Tesoriere R, Viganò L, Caggiano L, Sgotto E, Capussotti L. Preoperative biliary drainage increases infectious complications after hepatectomy for proximal bile duct tumor obstruction. World J Surg 2009;33(2):318-325
23 Lee SH, Park JK, Yoon WJ, et al. Optimal biliary drainage for inoperable Klatskin's tumor based on Bismuth type. World J Gastroenterol 2007;13(29):3948-3955

24 Bories E, Pesenti C, Caillol F, Lopes C, Giovannini M. Transgastric endoscopic ultrasonography-guided biliary drainage: results of a pilot study. Endoscopy 2007;39(4):287-291

25 Horaguchi J, Fujita N, Noda Y, et al. Endosonography-guided biliary drainage in cases with difficult transpapillary endoscopic biliary drainage. Dig Endosc 2009;21(4):239-244

26 Park DH, Koo JE, Oh J, et al. EUS-guided biliary drainage with onestep placement of a fully covered metal stent for malignant biliary obstruction: a prospective feasibility study. Am J Gastroenterol 2009;104(9):2168-2174

27 Wang AY, Strand DS, Shami VM. Prevention of post-ERCP pancreatitis: medications and techniques. Clin Gastroenterol Hepatol 2016; pii:S1542-3565(16)30215-4. doi: 10.1016/j.cgh.2016.05.026. [Epub ahead of print]

28 Patel IJ, Davidson JC, Nikolic B, et al; Standards of Practice Committee, with Cardiovascular and Interventional Radiological Society of Europe (CIRSE) Endorsement. Consensus guidelines for periprocedural management of coagulation status and hemostasis risk in percutaneous image-guided interventions. J Vasc Interv Radiol 2012;23(6):727-736

29 Wayne PH III, Whelan JG Jr. Susceptibility testing of biliary bacteria obtained before bile duct manipulation. AJR Am J Roentgenol 1983;140(6):1185-1188

30 Spies JB, Rosen RJ, Lebowitz AS. Antibiotic prophylaxis in vascular and interventional radiology: a rational approach. Radiology 1988;166(2):381-387

31 Speer AG, Cotton PB, Russell RC, et al. Randomised trial of endoscopic versus percutaneous stent insertion in malignant obstructive jaundice. Lancet 1987;2(8550):57-62

32 Becker CD, Glättli A, Maibach R, Baer HU. Percutaneous palliation of malignant obstructive jaundice with the Wallstent endoprosthesis: follow-up and reintervention in patients with hilar and non-hilar obstruction. J Vasc Interv Radiol 1993;4(5):597-604

33 Rossi P, Bezzi M, Rossi M, et al. Metallic stents in malignant biliary obstruction: results of a multicenter European study of 240 patients. J Vasc Interv Radiol 1994;5(2):279-285

34 Berkman WA, Bishop AF, Palagallo GL, Cashman MD. Transhepatic balloon dilation of the distal common bile duct and ampulla of Vater for removal of calculi. Radiology 1988;167(2): 453-455

35 Fidelman N, Bloom AI, Kerlan RK Jr, et al. Hepatic arterial injuries after percutaneous biliary interventions in the era of laparoscopic surgery and liver transplantation: experience with 930 patients. Radiology 2008;247(3):880-886

36 Mueller PR, vanSonnenberg E, Ferrucci JT Jr, et al. Biliary stricture dilatation: multicenter review of clinical management in 73 patients. Radiology 1986;160(1):17-22

37 Carrasco CH, Zornoza J, Bechtel WJ. Malignant biliary obstruction: complications of percutaneous biliary drainage. Radiology 1984; 152(2):343-346

38 Chapman WC, Sharp KW, Weaver F, Sawyers JL. Tumor seeding from percutaneous biliary catheters. Ann Surg 1989;209(6): 708-713, discussion 713-715

39 Audisio RA, Morosi C, Bozzetti F, et al. The outcome of cholangitis after percutaneous biliary drainage in neoplastic jaundice. HPB Surg 1993;6(4):287-293

40 Libby ED, Leung JW. Prevention of biliary stent clogging: a clinical review. Am J Gastroenterol 1996;91(7):1301-1308

41 Lorenz JM, Zangan SM, Leef JA, Van Ha TG. Iatrogenic portobiliary fistula treated by stent-graft placement. Cardiovasc Intervent Radiol 2010;33(2):421-424

42 Amikura K, Kobari M, Matsuno S. The time of occurrence of liver metastasis in carcinoma of the pancreas. Int J Pancreatol 1995; 17(2):139-146 
43 Kayahara M, Nagakawa T, Ueno K, Ohta T, Takeda T, Miyazaki I. An evaluation of radical resection for pancreatic cancer based on the mode of recurrence as determined by autopsy and diagnostic imaging. Cancer 1993;72(7):2118-2123

44 Yeoh KG, Zimmerman MJ, Cunningham JT, Cotton PB. Comparative costs of metal versus plastic biliary stent strategies for malignant obstructive jaundice by decision analysis. Gastrointest Endosc 1999;49(4, Pt 1):466-471

45 Wagner HJ, Knyrim K, Vakil N, Klose KJ. Plastic endoprostheses versus metal stents in the palliative treatment of malignant hilar biliary obstruction. A prospective and randomized trial. Endoscopy $1993 ; 25(3): 213-218$

46 Salomonowitz EK, Adam A, Antonucci F, Stuckmann G, Zollikofer CL. Malignant biliary obstruction: treatment with self-expandable stainless steel endoprosthesis. Cardiovasc Intervent Radiol 1992; 15(6):351-355

47 Rossi P, Bezzi M, Salvatori FM, Panzetti C, Rossi M, Pavia G. Clinical experience with covered wallstents for biliary malignancies: 23month follow-Up. Cardiovasc Intervent Radiol 1997;20(6):441-447

48 Freeman ML, Overby C. Selective MRCP and CT-targeted drainage of malignant hilar biliary obstruction with self-expanding metallic stents. Gastrointest Endosc 2003;58(1):41-49

49 Son RC, Gwon DI, Ko HK, Kim JW, Ko GY. Percutaneous unilateral biliary metallic stent placement in patients with malignant obstruction of the biliary hila and contralateral portal vein stenoocclusion. Korean J Radiol 2015;16(3):586-592

50 Gwon DI, Ko GY, Yoon HK, et al. Safety and efficacy of percutaneous Y-configured covered stent placement for malignant hilar biliary obstruction: a prospective, pilot study. J Vasc Interv Radiol 2012; 23(4):528-534

51 Jo JH, Park BH. Suprapapillary versus transpapillary stent placement for malignant biliary obstruction: which is better? J Vasc Interv Radiol 2015;26(4):573-582

52 Bakhru M, Ho HC, Gohil V, et al. Fully-covered, self-expandable metal stents (CSEMS) in malignant distal biliary strictures: midterm evaluation. J Gastroenterol Hepatol 2011;26(6):1022-1027

53 Hausegger KA, Thurnher S, Bodendörfer G, et al. Treatment of malignant biliary obstruction with polyurethane-covered Wallstents. AJR Am J Roentgenol 1998;170(2):403-408

54 Fanelli F, Orgera G, Bezzi M, Rossi P, Allegritti M, Passariello R. Management of malignant biliary obstruction: technical and clinical results using an expanded polytetrafluoroethylene fluorinated ethylene propylene (ePTFE/FEP)-covered metallic stent after 6-year experience. Eur Radiol 2008;18(5):911-919

55 Han YM, Kwak HS, Jin GY, Lee SO, Chung GH. Treatment of malignant biliary obstruction with a PTFE-covered self-expandable nitinol stent. Korean J Radiol 2007;8(5):410-417

56 Hatzidakis A, Krokidis M, Kalbakis K, Romanos J, Petrakis I, Gourtsoyiannis N. ePTFE/FEP-covered metallic stents for palliation of malignant biliary disease: can tumor ingrowth be prevented? Cardiovasc Intervent Radiol 2007;30(5):950-958

57 Schoder M, Rossi P, Uflacker R, et al. Malignant biliary obstruction: treatment with ePTFE-FEP- covered endoprostheses initial technical and clinical experiences in a multicenter trial. Radiology 2002;225(1):35-42

58 Krokidis M, Fanelli F, Orgera G, et al. Percutaneous palliation of pancreatic head cancer: randomized comparison of ePTFE/FEPcovered versus uncovered nitinol biliary stents. Cardiovasc Intervent Radiol 2011;34(2):352-361

59 Kullman E, Frozanpor F, Söderlund C, et al. Covered versus uncovered self-expandable nitinol stents in the palliative treatment of malignant distal biliary obstruction: results from a randomized, multicenter study. Gastrointest Endosc 2010; 72(5):915-923

60 Suk KT, Kim JW, Kim HS, et al. Human application of a metallic stent covered with a paclitaxel-incorporated membrane for malignant biliary obstruction: multicenter pilot study. Gastrointest Endosc 2007;66(4):798-803

61 Alis H, Sengoz C, Gonenc M, Kalayci MU, Kocatas A. Endobiliary radiofrequency ablation for malignant biliary obstruction. Hepatobiliary Pancreat Dis Int 2013;12(4):423-427

62 Dolak W, Schreiber F, Schwaighofer H, et al; Austrian Biliary RFA Study Group. Endoscopic radiofrequency ablation for malignant biliary obstruction: a nationwide retrospective study of 84 consecutive applications. Surg Endosc 2014;28(3): $854-860$

63 Lee MJ, Dawson SL, Mueller PR, et al. Failed metallic biliary stents: causes and management of delayed complications. Clin Radiol 1994;49(12):857-862

64 Lee TH, Park SH, Kim SP, et al. Chemical ablation of the gallbladder using alcohol in cholecystitis after palliative biliary stenting. World J Gastroenterol 2009;15(16):2041-2043 